\title{
ANÁLISE COMPARATIVA TEÓRICO-PRÁTICA ENTRE SOFTWARES DE DETECÇÃO DE PLÁGIO
}

\author{
Felipe Becker Nunes, PPGI, UFSM, nunesfb@gmail.com \\ Gleizer Bierhalz Voss, PPGI, UFSM, gleizer.voss@gmail.com \\ Andreia Rosangela Kessler Muhlbeier, PPGI, UFSM, andreiamuhlbeier@yahoo.com.br \\ Catiane Priscila Barbosa Arenhardt, PPGI, UFSM, catianepriscilabarbosa@gmail.com \\ Roseclea Duarte Medina, PPGI, UFSM, roseclea.medina@gmail.com \\ Érico M. H. Amaral, PPGIE/UFRGS, UNIPAMPA, ericohoffamaral@gmail.com \\ Giliane Bernardi, CCC, UFSM, giliane@inf.ufsm.br
}

Resumo. Concomitantemente ao crescimento das TICs, têm aumentado os casos de plágio, tanto no meio acadêmico quanto na área de comunicação/ jornalística, afetando os professores, alunos e demais profissionais. Este artigo apresenta um comparativo entre ferramentas de detecção de plágio com o objetivo de indicar quais destas permitem aos usuários efetuarem análises dos documentos de forma mais ágil, e ao mesmo tempo com resultados mais concisos. Os softwares avaliados foram o Farejador de Plágio, Miss Marple, Plagius Detector e Viper, nos quais foram aplicados testes de precisão de resultados, tempo de processamento e análise da interface, de forma a concluir quais apresentam melhores resultados em cada atributo analisado e no computo geral.

Palavras - Chave: plágio, softwares de detecção de plágio, estilometria.

\section{COMPARATIVE ANALYSIS BETWEEN THEORY-PRACTICE SOFTWARE FOR DETECTION OF PLAGIARISM}

\begin{abstract}
Concurrent with the growth of TICs have increased cases of plagiarism, both in academia and in the area of communication/journalism, affecting teachers, students and other professionals. This paper presents a comparative plagiarism detection tools in order to indicate which of these allow users log analysis of documents in a more agile, and at the same time with more concise results. The software evaluated were Sniffer Plagiarism, Miss Marple, and Viper Detector Plagius in which tests were used precision of results, time processing and analysis of the interface in order to conclude which show better results in every attribute analysis and computation general.
\end{abstract}

Keywords: plagiarism, detection software plagiarism, estilometria.

\section{INTRODUÇÃOO}

A evolução tecnológica nas últimas décadas tem facilitado a comunicação e a interação entre as pessoas e o mundo virtual, a qualquer hora e lugar. Do mesmo modo, a Internet transformou-se em um acervo de informações, na qual localizam-se os mais diversos conteúdos (e. g, trabalhos de conclusão de curso, dissertações, artigos e diversas outras formas de divulgação do conhecimento). Segundo Silva e Domingues (2008), a expansão da Internet com o acesso a diversos repositórios dos mais variados assuntos aumentou consideravelmente a incidência de plágio, principalmente no meio acadêmico.

Entretanto, esta prática não é restrita ao domínio da Internet, sendo que casos de plágio podem acontecer em meios não digitais, como livros e obras literárias. Contudo, 
o maior nível de preocupação é em relação aos arquivos digitais, devido à facilidade que o plagiador tem de encontrar informações relacionadas aos assuntos pesquisados e realizar uma simples e rápida cópia de textos, apropriando-se da criação de outras pessoas. De acordo com Pertile (2011), a prática de plágio em trabalhos acadêmicos vem se tornando um problema cada vez maior para os professores, pois os alunos têm realizado esta ação sem se preocupar com as consequências que isto pode causar.

Neste contexto, alternativas têm sido desenvolvidas para combater este tipo de ação, como as ferramentas para detecção de plágio, que realizam varreduras no documento com o objetivo de analisar nos principais motores de busca a existência de trechos semelhantes ao escrito no documento. Neste sentido, autores como Oliveira et al. (2007) evidenciam a carência de mecanismos de recuperação de informação mais eficazes para o controle de grandes volumes de informações, em particular para detecção de indícios de plágios.

Porém, a utilização dessas ferramentas pode se tornar uma tarefa complexa, quando desenvolvidas sem a utilização/observação de técnicas de IHC (Interação Humano-Computador), uma vez que a construção e o uso de um artefato ocorrem em contextos distintos e seguem lógicas diferentes, envolvendo pessoas diversas (Barbosa e da Silva (2010, p. 7). Além disso, a dificuldade da análise e a interpretação dos resultados contribuem de forma negativa no uso desses softwares de detecção de plágio. Para os autores "quem desenvolve tecnologia precisa sempre se perguntar: o que acontece se o usuário errar, a tecnologia falhar ou permanecer indisponível por algum tempo? As salvaguardas serão desenvolvidas de acordo com as respostas a perguntas como essa".

Este artigo apresenta um comparativo entre ferramentas de detecção de plágio com o objetivo de indicar quais permitem aos usuários efetuarem análises dos documentos de forma mais ágil, e ao mesmo tempo com resultados concisos. Para isso, foram analisadas suas principais funcionalidades, através da aplicação de um checklist de requisitos da engenharia de software, além da realização de testes práticos a partir da análise de documentos. As ferramentas selecionadas foram: Farejador de Plágio, Plagius Detector, Viper e Miss Marple.

\section{DETECÇÃo DE PLÁGIO}

Identificar o plágio não é simples, exige tempo e esforço de quem o procura. O mal uso da Internet faz com que circulem informações da multiplicação do índice de plágio em vários tipos de trabalhos acadêmicos, estes manifestados de diversas maneiras e classificados por inúmeras definições (Lima, 2011).

Para Oliveira et al. (2007), a definição de plágio é dada por um processo de apropriação ou imitação dos trabalhos alheios, não fornecendo o crédito necessário aos seus legítimos autores. De acordo com Rosales et al. (2008), o plágio é o ato de assinar uma obra de qualquer natureza contendo partes de uma outra pertencente a outro autor sem que sejam referenciadas a autoria da obra consultada.

Para Kang, Gelbukh e Han (2006) o nível de dificuldade de detecção de plágio em documentos de texto pode ser classificado conforme determinadas características de tipo de plágio. O plágio é identificado com maior facilidade em cópias na íntegra ou em parte de sua estrutura, conforme ilustra a Figura 1. 


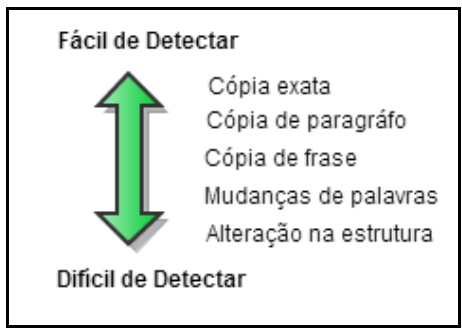

Figura 1 - Padrões de plágio e seus níveis.

Fonte: Adaptado de Kang, Gelbukh e Han (2006).

Conforme Maurer, Kappe e Zaka (2006), existem três maneiras de identificar o plágio através de softwares: Comparação entre documentos, no qual é realizada uma comparação entre um documento e um conjunto de documentos; a Busca por parágrafos na Internet, na qual a análise é executada por frases ou expressões suspeitas e a Estilometria, que analisa o estilo da escrita do texto por comparações de documentos de mesmo autor.

$\mathrm{Na}$ tentativa de diminuir as ocorrências de plágio, principalmente em trabalhos acadêmicos, diversas ferramentas são desenvolvidas, tanto on-line quanto desktop, para auxiliar na detecção destes casos. As ferramentas on-line rodam diretamente no navegador, dentre as quais citam-se o Approbo, iThenticate, Plagiarism e Turnitin. Quanto aos desktops, que são instalados diretamente no computador, pode-se citar o Farejador de Plágio, JPlag, Miss Marple, Plagius Detector e Viper (Pertile, 2011).

Neste contexto, para o processo de detecção, utilizam-se softwares automatizados que identificam similaridade entre documentos, através de trechos de texto ou de código. Dentre estes foram selecionados os softwares: Farejador de Plágio, Plagius Detector, Viper e Miss Marple. A escolha das ferramentas utilizadas nos testes foi realizada após uma pesquisa bibliográfica em trabalhos como: (Lima, 2011) e (Pertile, 2011), em que foram analisadas as principais funcionalidades disponíveis.

\subsection{Farejador de Plágio (FDP)}

Farejador de Plágio é uma ferramenta que realiza a análise de documentos com base na leitura de trechos contínuos de texto em sites de busca. Ao finalizar as buscas, são aplicadas diversas técnicas de processamento de dados a fim de realizar o apontamento do que é ou não copiado da internet (Farejador, 2012).

\subsection{Plagius Detector de Plágio}

É um software pago (possui versão gratuita para testes) que permite analisar documentos na íntegra, na busca de indícios de plágio. Esta ferramenta permite gerar relatórios com detalhes da busca realizada, retornando inclusive o percentual de suspeitas encontradas. Além disso, oferece um plug-in para o MS Word 2007 capaz de verificar o plágio no momento da digitação. As principais vantagens apontadas pelos desenvolvedores da ferramenta são: a simplificidade de operação, desempenho, rapidez e eficiência (Plagius, 2012).

\subsection{Viper}


Esta ferramenta realiza buscas na Internet a partir de algumas palavras-chave comparando-as com trechos do texto submetido para análise (Viper, 2012). Desenvolvida pela Scanmyessay, e distribuída de forma gratuita, no entanto este software retém uma cópia do arquivo analisado durante um período de nove meses, podendo ser utilizada por outros usuários para consultas futuras.

\subsection{Miss Marple}

Este software foi criado a partir do método Detector de Indícios de Plágio (DIP) (Pertile e Medina, 2011), sendo desenvolvido com uma API de buscas do Google - API Google Search Ajax, onde os arquivos são enviados para análise e comparação e o cálculo de similaridade é feito em relação ao content retornado da pesquisa, levando em consideração a indexação dos termos com a utilização da biblioteca Lucene - Java. Essa biblioteca possibilita a aplicação de técnicas de stemming ao indexar termos, facilitando a comparação entre termos de mesmo radical, por exemplo: Carro e Carroça.

\section{METODOLOGIA}

A confiabilidade dos relatórios gerados por estes softwares é uma questão muito importante em relação ao processo de auxiliar o avaliador na determinação de um caso de plágio. Para isto, o usuário deve estar ciente da escolha da ferramenta a ser utilizada neste processo, quais as vantagens e desvantagens que ela possui, possibilitando detectar de forma eficiente a cópia de trechos no texto.

Os testes realizados buscaram comparar três dos principais aspectos que uma ferramenta de detecção deve possuir: a interface de utilização, o tempo de processamento da análise e a precisão dos resultados apresentados sobre os documentos. Além disso, os demais testes e estudos baseiam-se na comparação das ferramentas escolhidas para detecção de plágio, identificando em um quadro comparativo os principais recursos que cada uma delas disponibiliza.

Nestas verificações foi utilizada uma máquina portando o sistema operacional Microsoft Windows XP Service Pack 3 com as seguintes configurações: processador Intel Pentium T4300, 4 GB de memória e 320 GB de armazenamento. Os testes foram realizados em duas conexões de banda larga com velocidades diferentes, de $1 \mathrm{Mbps} \mathrm{e}$ $10 \mathrm{Mbps}$.

Os avaliadores que realizaram os testes nas ferramentas detêm o perfil da área da computação, ou seja, possuem conhecimentos avançados em informática, possibilitando assim a análise de precisão dos resultados obtidos, do tempo de processamento e avaliação da interface das ferramentas de detecção.

Para a realização dos testes foram utilizados cinco tipos diferentes de documentos, todos com um tamanho fixo de quatro páginas, que foram elaborados da seguinte forma:

- Documento 01: o texto foi totalmente plagiado com trechos de artigos publicados em congressos e periódicos. As citações utilizadas são de autores aleatórios, mesclando assim os parágrafos do texto.

- Documento 02: o texto possui três páginas totalmente plagiadas e uma sem nenhuma semelhança com artigos publicados na Internet.

- Documento 03: o texto possui duas páginas totalmente plagiadas e duas sem nenhuma semelhança com artigos publicados na Internet. 
- Documento 04: o texto possui uma página totalmente plagiada e três sem nenhuma semelhança com artigos publicados na Internet.

- Documento 05: o texto possui todas páginas sem nenhuma semelhança com artigos publicados na Internet, ou seja, não possui nenhum indicio de plágio.

\subsection{Comparativo das ferramentas analisadas}

Com base nas pesquisas bibliográficas realizadas a respeito dos quatro softwares analisados, além dos testes realizados nos mesmos, foi elaborado uma tabela comparativa dos principais recursos disponíveis nas ferramentas. A Tabela 1 apresenta o comparativo realizado.

Tabela 1 - Comparativo entre as ferramentas analisadas.

\begin{tabular}{l|c|c|c|c}
\hline Ferramentas / Requisitos & Farejador de Plágio & Miss Marple & Plagius Detector & Viper \\
\hline $\begin{array}{l}\text { Tipos de extensões suportadas } \\
\text { (.doc; .docx; .pdf; .odt; .rtf) }\end{array}$ & .doc; .docx; .rtf; & $\begin{array}{c}\text {.doc; .docx; } \\
\text {.pdf; }\end{array}$ & $\begin{array}{c}\text {.doc; .docx; .pdf; } \\
\text {.odt; .rtf }\end{array}$ & .doc; .docx \\
\hline Plataformas suportadas & Windows & $\begin{array}{c}\text { Windows; } \\
\text { Linux }\end{array}$ & Windows & Windows \\
\hline Licença do software & Pago* & Gratuito & Pago* & Gratuito \\
\hline $\begin{array}{l}\text { Permite configurar profundidade } \\
\text { da análise }\end{array}$ & Sim & Não & Sim & Sim \\
$\begin{array}{l}\text { Gravação dos resultados de } \\
\text { análise em arquivo }\end{array}$ & Sim & Sim & Sim & Sim \\
\hline
\end{tabular}

* Possuem versões gratuitas, porém com limitações no tamanho do documento a ser analisado. No entanto, para a realização deste trabalho foram utilizadas as versões completas das respectivas ferramentas.

Na tabela 1 é possível visualizar que a ferramenta Plagius Detector suporta um maior número de formatos de arquivos para análise em relação às outras, fornecendo assim mais alternativas aos usuários. As plataformas suportadas pelas ferramentas são em geral Windows, com exceção do Miss Marple, que também pode ser instalado no Linux.

Todas gravam os resultados da análise do documento em um arquivo. Quanto as configurações das ferramentas, com exceção do Miss Marple, todas permitem realizar a seleção da profundidade da pesquisa, que envolve o intervalo de palavras a serem analisadas e outros aspectos.

\section{ANÁLISE DOS RESULTADOS}

Após a realização dos testes nas quatro ferramentas, nos quais foram analisadas interfaces, tempo de processamento e precisão dos resultados, realizou-se um estudo dos dados coletados com o objetivo de identificar os pontos positivos e negativos das ferramentas em cada aspecto analisado. Além disso, após as verificações, foi definida qual delas é a mais indicada para a detecção de plágio.

\subsection{Interface de utilização}

A interface de cada ferramenta utilizada difere das demais, o que faz com que seja necessária a avaliação de alguns aspectos relacionados à operação das mesmas por parte dos usuários. Para a realização deste processo foi elaborado um checklist, ou seja, uma 
lista de verificação para que após a utilização das ferramentas seja feita sua avaliação pelos usuários. O checklist foi adaptado de duas fontes: o questionário ErgoList ${ }^{\prime}$ e a norma ISO/IEC $9126^{2}$.

Na Tabela 2, é apresentada a avaliação realizada pelo cálculo da média de respostas dos três avaliadores, com a utilização da tecnologia do Google Forms, que cria formulários com múltiplas questões fornecendo dados estatísticos das mesmas. As questões são respondidas de forma objetiva distribuídas em escala com três alternativas de resposta: Sim, Parcialmente e Não.

Tabela 2 - Questões de avaliação das ferramentas de softwares de detecção de plágio.

\begin{tabular}{|c|c|c|c|c|}
\hline Ferramentas / Perguntas & $\begin{array}{l}\text { Farejador de } \\
\text { Plágio } \\
\end{array}$ & Miss Marple & Plagius Detector & Viper \\
\hline $\begin{array}{l}\text { O software dispõe de todas as } \\
\text { funções necessárias para a execução? }\end{array}$ & Sim & Sim & Sim & $\operatorname{Sim}$ \\
\hline $\begin{array}{l}\text { 2) É permitida a exportação dos } \\
\text { dados da análise? }\end{array}$ & Sim & Sim & Sim & $\operatorname{Sim}$ \\
\hline $\begin{array}{l}\text { 3) Apresenta falhas com } \\
\text { frequência? }\end{array}$ & Parcialmente & Não & Não & Parcialmente \\
\hline $\begin{array}{l}\text { 4) O objeto é conciso nos } \\
\text { resultados, passando confiança ao } \\
\text { usuário? }\end{array}$ & Parcialmente & Parcialmente & Sim & Parcialmente \\
\hline $\begin{array}{l}\text { 5) Os trechos indicados de } \\
\text { plágio estão corretos? }\end{array}$ & Parcialmente & Parcialmente & Sim & Parcialmente \\
\hline $\begin{array}{l}\text { 6) As referências apresentadas } \\
\text { pelo sistema estão de acordo com os } \\
\text { trechos copiados? }\end{array}$ & Parcialmente & Parcialmente & Sim & Parcialmente \\
\hline $\begin{array}{l}\text { 7) As indicações de plágio no } \\
\text { texto são concisas? }\end{array}$ & Parcialmente & Parcialmente & Sim & Parcialmente \\
\hline O objeto é de fácil utilização? & Parcialmente & Sim & Sim & Sim \\
\hline É fácil de aprender a usar? & Sim & Sim & Sim & Sim \\
\hline $\begin{array}{l}\text { 10) Os arquivos de instalação } \\
\text { funcionam corretamente? }\end{array}$ & Sim & Parcialmente & Sim & Parcialmente \\
\hline $\begin{array}{l}\text { 11) Todos os campos e } \\
\text { mostradores de dados possuem rótulos } \\
\text { identificativos? }\end{array}$ & Sim & Sim & Sim & Sim \\
\hline $\begin{array}{l}\text { 12) Caso o arquivo a ser analisado } \\
\text { possua um formato específico (PDF, } \\
\text { DOCX, DOC), este formato encontra- } \\
\text { se descrito? }\end{array}$ & Parcialmente & Sim & Parcialmente & Não \\
\hline $\begin{array}{l}\text { 13) O sistema fornece ao usuário } \\
\text { informações sobre o tempo de } \\
\text { processamento? }\end{array}$ & Parcialmente & Não & Sim & Sim \\
\hline $\begin{array}{l}\text { 14) O usuário encontra disponível } \\
\text { as informações necessárias para suas } \\
\text { ações através do botão de comando } \\
\text { "AJUDA"? }\end{array}$ & Sim & Não & Sim & Parcialmente \\
\hline $\begin{array}{l}\text { 15) Na ocorrência de erros, o } \\
\text { usuário pode acessar todas as } \\
\text { informações necessárias ao diagnóstico } \\
\text { e à solução do problema? }\end{array}$ & Parcialmente & Não & Parcialmente & Parcialmente \\
\hline $\begin{array}{l}\text { 16) Quando, durante a análise do } \\
\text { documento, o sistema torna-se } \\
\text { indisponível ao usuário, devido a } \\
\text { algum processamento longo, este é }\end{array}$ & Sim & Parcialmente & Sim & Parcialmente \\
\hline
\end{tabular}


avisado desse estado do sistema e do tempo dessa indisponibilidade?

Na tabela 2 é possível visualizar que nas questões 4, 5, 6 e 7, somente o Plagius Detector obteve resultados com um grau de precisão alto, sendo que as outras ferramentas apresentaram resultados com falhas de consistência. Estas falhas foram a não marcação de indícios de plágio em trechos copiados dos autores, assim como a disponibilização de links incorretos com o trecho verificado. Outra observação que pode ser realizada é em relação à questão 12, em que somente a ferramenta Miss Marple apresentou a opção de escolha do tipo de arquivo a ser pesquisado, enquanto as demais apresentavam os tipos de arquivos de forma geral para os usuários.

Com relação à interface das ferramentas, o Plagius Detector obteve a melhor classificação por parte dos avaliadores, sendo considerada sua interface intuitiva e clara, atendendo aos requisitos necessários, descritos no checklist, para que exista uma boa usabilidade pelos usuários. O Farejador de Plágio e o Viper contam com uma interface mais simples, não disponibilizando todos os atributos necessários para que haja uma utilização sem problemas pelos usuários. Por fim, a ferramenta Miss Marple obteve a avaliação mais baixa por parte dos usuários que responderam ao questionário, devido à falta de maiores informações e recursos de auxílio ao usuário, tornando-a excessivamente simplista.

\subsection{Tempo de processamento da análise}

O tempo de processamento da análise foi calculado para cada ferramenta através da média obtida dos três testes realizados pelos usuários. Cada ferramenta possui um cronômetro integrado que fornece o tempo decorrido para a realização da análise. As Figuras 2 e 3 apresentam os resultados obtidos.

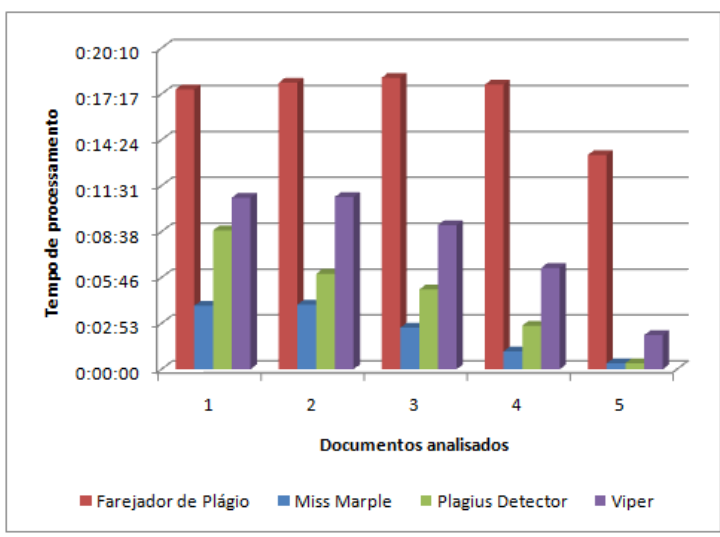

Figura 2 - Tempo de processamento com velocidade de $1 \mathrm{Mbps}$.

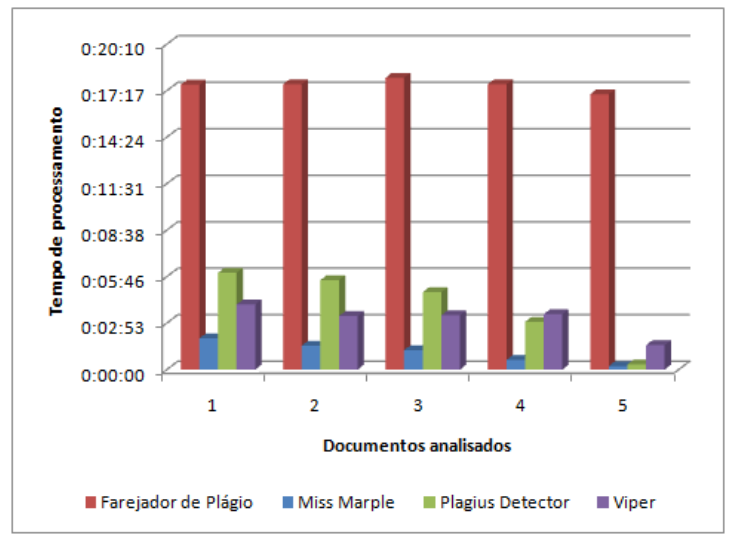

Figura 3 - Tempo de processamento com velocidade de $10 \mathrm{Mbps}$.

Quanto ao tempo de processamento das análises, no comparativo realizado entre as quatro ferramentas, é possível perceber que o software Miss Marple atingiu os melhores tempos de processamento nas duas velocidades testadas, destacando-se em relação às outras ferramentas neste atributo. Já o Farejador de Plágio obteve os piores resultados, tornando-o a ferramenta com maior tempo de processamento em relação às analisadas. 
Pode-se observar nas figuras 2 e 3 que o Farejador de Plágio teve unanimidade quanto ao tempo de processamento, sendo o mais lento dentre todas ferramentas analisadas, independente do arquivo ou conexão em que foi testado. Também percebese que a ferramenta Miss Marple obteve o mesmo comportamento, mas de maneira inversa, sendo que obteve os tempos de processamento mais rápidos, indiferente da conexão ou documento em que foi testado.

Nos testes realizados em uma conexão com velocidade de 1Mbps, o Miss Marple obteve os menores tempos de processamento, enquanto o Plagius Detector e Viper atingiram o segundo e terceiro melhor tempo respectivamente. Por fim, o Farejador de Plágio foi a ferramenta que obteve os piores resultados, com tempos mais altos do que a média, tornando-o a ferramenta com o pior tempo de processamento.

Nos testes utilizando uma conexão com velocidade de $10 \mathrm{Mbps}$, ocorreram mudanças significativas no tempo de processamento em três das ferramentas testadas, sendo que o Farejador de Plágio continuou sendo a ferramenta com os tempos de processamento mais elevados em relação às demais analisadas. O Plagius Detector realizou a análise dos documentos com um tempo médio maior do que o Viper, diferentemente do ocorrido com a velocidade de $1 \mathrm{Mbps}$, atingindo o terceiro melhor tempo, alterando a posição com o Viper, que obteve a segunda colocação nesse quesito. Já o Miss Marple manteve os tempos mais baixos de processamento na realização das análises.

\subsection{Precisão dos resultados apresentados}

A análise do percentual de precisão dos resultados foi realizada de forma manual, na qual foi calculado a média dos resultados obtidos após a verificação de cada referência que tenha sido apontada como plágio, identificando o número total de referências existentes no texto e o total destas que foram apontadas a possibilidade de plágio. Foram realizados os testes em duas velocidades de conexão diferentes devido ao fato de que todas as ferramentas realizam buscas na Internet para identificar possíveis trechos de plágio, tendo a possibilidade de ocorreram variações na precisão dos resultados devido à velocidade, por exemplo, por não conseguir realizar o download de um arquivo com tamanho muito grande.

O percentual de profundidade da verificação dos documentos definido em todas as ferramentas foi de $80 \%$. Por exemplo, no Documento 01 que está totalmente plagiado existem 25 parágrafos, em que cada um destes é uma citação plagiada de um artigo. Assim, se a ferramenta apontar 13 parágrafos contendo plágio, sua precisão será de $50 \%$, já que identificou somente metade dos trechos plagiados no documento. As Figuras 4 e 5 apresentam os resultados obtidos em uma conexão de $1 \mathrm{Mbps}$ e $10 \mathrm{Mbps}$ respectivamente. 


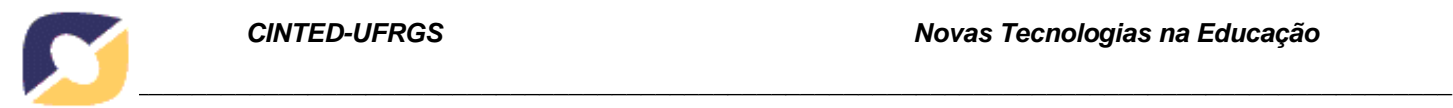

9

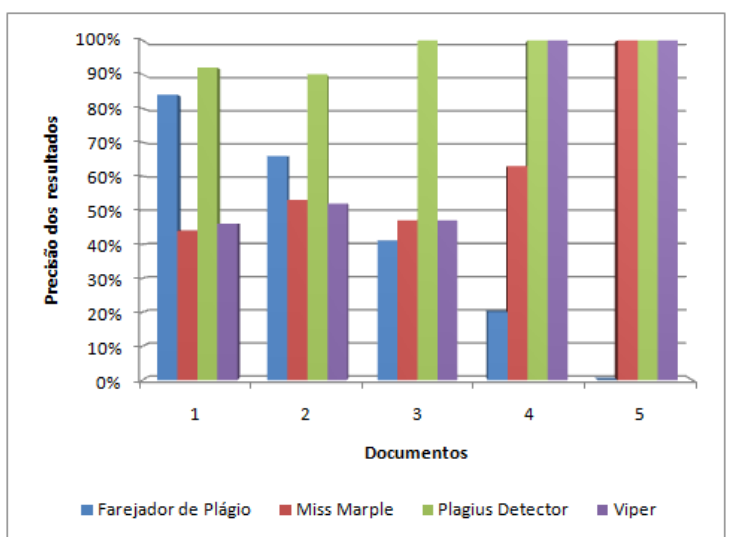

Figura 4 - Precisão dos resultados com velocidade de $1 \mathrm{Mbps}$.

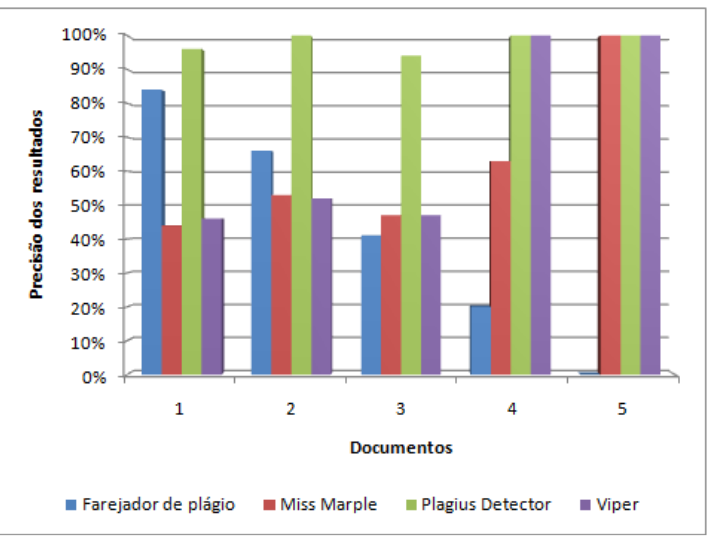

Figura 5 - Precisão dos resultados com velocidade de $10 \mathrm{Mbps}$.

Para finalizar, a análise de precisão dos resultados, pode-se perceber que não ocorreram alterações de precisão no resultado das ferramentas quando testadas em conexões com velocidade diferentes (1 Mbps e $10 \mathrm{Mbps}$ ). Após a verificação dos resultados obtidos nos cincos documentos por cada ferramenta, nota-se que o Plagius Detector atingiu uma porcentagem significativa de precisão, com uma média de $98 \%$ de precisão em uma velocidade de $10 \mathrm{Mbps}$ e $96,4 \%$ com $1 \mathrm{Mbps}$, o que significa que ela obteve o maior número de acertos em relação aos trechos que possuíam plágio e aos que não tinham. O Farejador de Plágio e o Viper atingiram precisões médias semelhantes, em que o primeiro obteve resultados concisos nos documentos 01 e 02 , enquanto que o segundo alcançou melhores resultados nos documentos 04 e 05 . Vale ressaltar que a ferramenta Farejador de Plágio detectou indícios de plágio em todos os trechos do documento cinco, sendo que este não possuía nenhum parágrafo contendo tal ação, ou seja, indicando falso positivo em todos os parágrafos analisados. Ressalta-se que as demais ferramentas não acusaram plágio em nenhum trecho do documento cinco, obtendo assim uma precisão de $100 \%$ ao não indicar absolutamente nada de plágio.

Portanto, após o estudo e as comparações realizadas, constatou-se que a ferramenta que mais se aproxima do esperado de um software de detecção de plágio de forma geral, na qual busca-se analisar documentos de forma mais ágil, e ao mesmo tempo com resultados concisos, além de uma interface de boa usabilidade, é o Plagius Detector.

\section{CONSIDERAÇÕES FINAIS}

Com o crescimento do número de casos de plágio no meio acadêmico, a utilização de ferramentas para análise deste tipo de ação tornou-se muito relevante, como forma de auxiliar professores e alunos a identificarem possíveis casos, amenizando os problemas que têm sido causados por esta prática. Com base nisso, verificou-se a necessidade da realização de um comparativo entre as ferramentas, de forma a orientar os usuários no momento da escolha.

As ferramentas de detecção de plágio estudadas neste trabalho foram: Farejador de Plágio, Miss Marple, Plagius Detector e Viper. Este artigo teve como objetivo realizar um comparativo entre estas quatro ferramentas, para identificar os principais pontos negativos e positivos de cada uma, de acordo com os requisitos analisados, 
apontando a ferramenta que mais se adéqua ao cumprimento da análise de detecção de plágio.

Neste sentido, a ferramenta Plagius Detector obteve os melhores resultados no âmbito geral da pesquisa, sendo esta considerada a mais adequada para a realização do processo de detecção de plágio. Ela foi considerada a mais precisa nos resultados analisados, além de possuir a melhor avaliação da interface. Também possui um tempo de processamento rápido, obtendo a segunda melhor média, perdendo somente para a ferramenta Miss Marple que atingiu os menores tempos de processamento.

Como trabalhos futuros, pretende-se aumentar o âmbito da pesquisa, incluindo um número maior de ferramentas a serem analisadas, além de adicionar outros aspectos relevantes na avaliação das ferramentas, como desempenho de Hardware e Rede. Além disso, realizar testes com documentos de maior extensão, aumentando o nível de exigência das ferramentas no processo de análise de detecção plágio.

${ }^{1}$ Disponível em: <http://www.labiutil.inf.ufsc.br/ergolist>.

2 Disponível em: <http://www.abntcatalogo.com.br/norma.aspx?ID=2815>.

\section{REFERÊNCIAS BIBLIOGRÁFICAS}

BARBOSA, S. D. J.; SILVA, B. S. da. Interação humano-computador. Editora Elsevier, pág. 7, 2010.

FAREJADOR. Site da ferramenta. 2012. Apresenta informações sobre a ferramenta, opções de download e guias de utilização. Disponível em: <http://www.farejadordeplagio.com.br/>. Acesso em: 21 out. 2012.

KANG, N.; GELBUKH, E.; HAN, S. PPChecker: Plagiarism Pattern Checker in Document Copy Detection. In: SOJKA, P.; KOPECEK, I.; PALA, K.Text, Speech and Dialogue: Springer Berlin / Heidelberg, v. 4188, 2006. p. 661-667.

LIMA, E. C. de. Análise de Técnica e Ferramentas de Detecção de Plágio, e Desenvolvimento de um Protótipo de Nova Ferramenta. Monografia de Conclusão de Curso - Universidade Federal de Lavras, Minas Gerais, 2011.

MAURER, H.; KAPPE, F.; ZAKA, B. Plagiarism - A Survey. Journal of Universal Computer Science, v. 12, n. 8, p. 1050-1084, Agosto 2006.

MISS MARPLE. Site do grupo que desenvolve a ferramenta. 2012. Apresenta informações sobre a ferramenta, opções de download e guias de utilização. Disponível em: 〈http://www.ufsm.br/greca>. Acesso em: 10 out. 2012.

NBR ISO/IEC 9126-1: 2003. Tecnologia de informação: Engenharia de software Qualidade de produto. Parte 1: Modelo de qualidade. Esta norma cancela e substitui a NBR 13596. Julho 2003.

OLIVEIRA, M.; PEREIRA, F.; CIARELLI, P. M.; CARDOSO, B.; HENRIQUE, W. F.; VERONESE, L.; OLIVEIRA, E. Bibliotecas digitais aliadas na detecção automática de plágio. V Simpósio Internacional de Bibliotecas Digitais, São Paulo, 2007.

PERTILE, S. L.; MEDINA, R. D. Desenvolvimento e Aplicação de um Método para Detecção de Indícios de Plágio. In: Simpósio Brasileiro de Informática na Educação, 2011, Aracajú. Anais do XXII SBIE - XVII WI, (2011). p. 1673-1682.

PERTILE, S. L. Desenvolvimento e aplicação de um método para detecção de indícios de plágio. Santa Maria, 2011, 72f. Dissertação de Mestrado em Informática Universidade Federal de Santa Maria (UFSM). 2011. 
PLAGIARISM DETECT. Services Plagiarism Detect: The Power of Uniqueness. Disponivel em: <http://www.plagiarismdetect.com/services.html>. Acesso em: 27 set. 2012.

PLAGIUS. Site da ferramenta. 2012. Apresenta informações sobre a ferramenta, opções de download e guias de utilização. Disponível em: <http://www.plagius.com/s/en/default.aspx>. Acesso em: 25 set. 2012.

ROSALES, F.; GARCIA, A.; RODRIGUES, S.; PEDRAZA, J.L.; MENDEZ, R.; NIETO, M. M.; Detection of plagiarism in programming assignments. IEEE Transactions on Education, v. 51, n. 2, p. 174-183, maio 2008.

SILVA, A. K. L. D.; DOMINGUES, M. J. C. D. S. Plágio no Meio Acadêmico: percepção dos alunos de pós-graduação sobre o tema. VI Simpósio de Gestão e Estratégia em Negócios, Seropédica, RJ, 2008.

VIPER. Site da ferramenta. 2012. Apresenta informações sobre a ferramenta, opções de download e guias de utilização. Disponível em: 〈http://www.scanmyessay.com px >. Acesso em: 10 out. 2012. 